\title{
Inhibition of Histone Deacetylases Prevents Cardiac Remodeling After Myocardial Infarction by Restoring Autophagosome Processing in Cardiac Fibroblasts
}

\author{
Yaping Wang ${ }^{\mathrm{a}}$ Panpan Chen ${ }^{\mathrm{a}} \quad$ Lihan Wang $^{\mathrm{a}} \quad$ Jing Zhao ${ }^{\mathrm{b}} \quad$ Zhiwei Zhong $^{\mathrm{b}}$ \\ Yingchao Wang ${ }^{\mathrm{b}}$ Jifeng $\mathrm{Xu}^{\mathrm{c}}$ \\ aDepartment of Cardiology, Second Affiliated Hospital Zhejiang University School of Medicine, \\ Hangzhou, bProvincial Key Laboratory of Cardiovascular Research, Hangzhou, 'Department of \\ Orthopaedic Surgery, Zhejiang Provincial People's Hospital, People's Hospital of Hangzhou Medical \\ College, Hangzhou, China
}

\section{Key Words}

TSA $•$ Cardiac remodeling • Autophagy

\begin{abstract}
Background/Aims: Histone deacetylases (HDACs) play a critical role in the regulation of gene transcription, cardiac development, and diseases. The aim of this study was to investigate whether the inhibition of HDACs improves cardiac remodeling and its underlying mechanisms in a mouse myocardial infarction (MI) model. Methods: The HDAC inhibitor trichostatin A (TSA, $0.1 \mathrm{mg} / \mathrm{kg} /$ day) was administered via daily intraperitoneal injections for 8 consecutive weeks after MI in C57/BL mice. Echocardiography and tissue histopathology were used to assess cardiac function. Cultured neonatal rat cardiac fibroblasts (NRCFs) were subjected to simulated hypoxia in vitro. Autophagic flux was measured using the tandem fluorescent mCherry-GFP-LC3 assay. Western blot was used to detect autophagic biomarkers. Results: After 8 weeks, the inhibition of HDACs in vivo resulted in improved cardiac remodeling and hence better ventricular function. MI was associated with increased LC3-II expression and the accumulation of autophagy adaptor protein p62, indicating impaired autophagic flux, which was reversed by TSA treatment. Cultured NRCFs exhibited increased cell death after simulated hypoxia in vitro. Increased cell death was associated with markedly increased numbers of autophagosomes but not autolysosomes, as assessed by punctate dual fluorescent mCherrygreen fluorescent protein tandem-tagged light chain-3 expression, indicating that hypoxia resulted in impaired autophagic flux. Importantly, TSA treatment reversed hypoxia-induced impaired autophagic flux and led to a $40 \%$ decrease in cell death. This was accompanied by improved mitochondrial membrane potential. The beneficial effects of TSA therapy were abolished by RNAi intervention targeting LAMP2; likewise, in vivo delivery of chloroquine
\end{abstract}




\section{Cellular Physiology Cell Physiol Biochem 2018;49:1999-2011

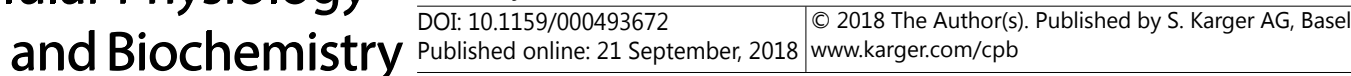 \\ Wang et al.: TSA and Cardiac Remodeling}

abolished the TSA-mediated cardioprotective effects. Conclusion: Our results provide evidence that the HDAC inhibitor TSA prevents cardiac remodeling after MI and is dependent on restoring autophagosome processing of cardiac fibroblasts.

\section{Introduction}

Adverse cardiac remodeling accompanied by enhanced cardiac fibrosis results in the progression of heart failure after myocardial infarction (MI) [1, 2]. The fibrosis that occurs during cardiac remodeling is due to an imbalance between the synthesis and degradation of the extracellular matrix (ECM) [3]. Cardiac fibroblasts (CFs), the predominant cell type in the heart, play a key role in the homeostatic maintenance of the ECM [4]. Thus, activated CFs play a key role in the adverse myocardial remodeling after MI and have become an important therapeutic target.

Chromatin remodeling enzymes are triggered during pathological cardiac remodeling. Acetylation and deacetylation of histone proteins are controlled by histone acetyltransferases and histone deacetylases (HDACs), respectively, which regulate the expression of target genes via the relaxation of chromatin and enhanced accessibility to DNA-binding proteins $[5,6]$. Small-molecule inhibitors of HDACs are currently being tested for a variety of oncological indications. Recently, suberoylanilide hydroxamic acid and romidepsin (Istodax), a depsipeptide HDAC inhibitor, gained US Food and Drug Administration approval for cutaneous T-cell lymphoma. Currently, there are more than 100 studies exploring the utility of this class of drugs for a variety of malignancies (www.clinicaltrials.gov). Gene deletion and overexpression studies have revealed important functions among several of these enzymes in various pathological cardiac remodeling processes [7-12]. However, the cellular target or targets-especially in CFs - of these powerful agents against disease progression are largely unknown.

Macroautophagy, also called autophagy, is a tightly regulated intracellular catabolic process that serves as a cellular quality control mechanism for the disposal of damaged and dysfunctional organelles and protein aggregates; thus, it is widely implicated in pathophysiological processes, including cardiovascular diseases $[13,14]$. The interruption of autophagy may lead to severe energy metabolism disorder and cell death [15]. Previous studies have suggested that autophagy is activated during various pathological conditions of the heart, such as MI, hypertrophy, and heart failure [16-18]. Autophagy has been clarified in myocardial ischemia/reperfusion settings, and it is believed that it plays distinct roles in the heart during ischemia and reperfusion. The induction of autophagy in the ischemic phase has a protective effect, whereas reperfusion-stimulated autophagy is implicated in cardiomyocyte death due to impaired autophagosome clearance [19-21]. However, the functional roles of autophagy in longer term MI remain unclear. A previous study has shown that autophagy triggered by long-term ischemia could be a homeostatic mechanism [22]. HDAC inhibition in a transverse aortic constriction (TAC) model showed profound suppression of load-induced cardiomyocyte autophagy, which could attenuate cardiac hypertrophy [23]. Other studies have demonstrated that HDACi reverses pre-existing systolic dysfunction and myocyte hypertrophy by blunting autophagy $[23,24]$. However, it is unclear whether and how HDACi regulates autophagy, especially in CFs during longer term MI.

To address these issues, we designed this study to (i) investigate whether TSA has cardioprotective effects on MI-induced autophagy dysfunction; (ii) clarify the effects of TSA on autophagy in CFs during MI; and (iii) determine the involvement of autophagosome clearance in CFs in TSA-mediated cardioprotective effects. Our results provide new insight into the mechanism of TSA-induced cardioprotection and suggest the potential value of TSA in protection of the heart against MI injury. 


\section{Cellular Physiology Cell Physiol Biochem 2018;49:1999-2011

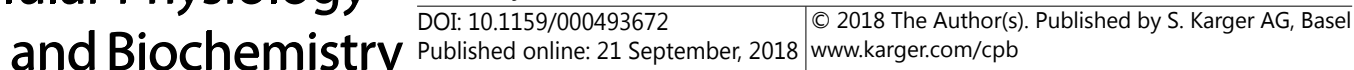

\section{Materials and Methods}

\section{Animals}

The animal study protocols complied with the National Institutes of Health (NIH) Guide for the Care of Use of Laboratory Animals (NIH Pub. No. 85-23, Revised 1996) and the Chinese Guidelines for the Care and Use of Laboratory Animals and were approved by the Zhejiang University Animal Care Committee.

\section{In vivo MI model}

Wild-type mice (C57BL/6, 8-10-week old, male, 20-25 g) were anesthetized by intraperitoneal injection of $4 \%$ chloral hydrate $(4 \mathrm{mg} / \mathrm{kg}$ ) and ventilated via tracheal intubation connected to a rodent ventilator. MI was induced by ligation of the left anterior descending (LAD) coronary artery using an 8-0 nylon suture. Animals were divided into three groups: 1) sham, where animals underwent thoracotomy without MI (without ligation); 2) MI, where animals underwent MI and received daily intraperitoneal injections of phosphate-buffered saline (PBS; $0.1 \mathrm{~mL}$ ) for 28 days thereafter as the control; and 3) MI+TSA, where animals that underwent MI received daily intraperitoneal injections of TSA $(0.1 \mathrm{mg} / \mathrm{kg}$; SigmaAldrich, St Louis, MO) for 8 weeks thereafter. Ventricular function was evaluated, hearts were harvested, and the infarcted left ventricles (LVs) were used for subsequent experiments.

\section{Cardiac function assessment by echocardiography}

At 8 weeks post-MI, all animals were anesthetized and underwent transthoracic parasternal short axis M-mode echocardiogram examination using a Vevo 2100 system (Visual Sonics, Toronto, Canada; http:// www.visualsonics.com/). Once a standard cross-section at the papillary muscle level was obtained, M-mode echocardiography was acquired and the images were stored. Fractional shortening (FS), ejection fraction (EF), left ventricular internal diastolic diameter (LVIDd), left ventricular internal systolic diameter (LVIDs), left ventricular diastolic posterior wall thickness (LVPWd), left ventricular systolic posterior wall thickness (LVPWs), interventricular septum thickness at end-diastole (IVSd), and interventricular septal thickness at end-systole (IVSs) were then analyzed for at least five consecutive cardiac cycles and the mean values were calculated.

\section{Histology and immunofluorescence}

Whole hearts were harvested and fixed with $2 \%$ paraformaldehyde at $41^{\circ} \mathrm{C}$ for $24 \mathrm{~h}$. Fixed tissues were dehydrated and embedded in paraffin, and 4- $\mu \mathrm{M}$-thick sections were prepared. Masson's trichrome staining was performed using standard methods. For immunofluorescence, sections were washed in PBS and blocked in 5-10\% donkey serum. Sections were incubated with antibodies to P62 (Abcam, Cambridge, UK) and vimentin (Cell Signaling Technology, Danvers, MA) followed by the appropriate secondary antibodies. Samples were viewed using a TCS SP2 confocal laser scanning microscope (Leica Microsystems, Wetzlar, Germany).

Neonatal rat ventricular fibroblast isolation, culture, and characterization

Primary cultures of neonatal rat ventricular fibroblasts (NRCFs) were isolated from hearts of 1-2-dayold Sprague-Dawley rats by trypsin and collagenase digestion as described previously [25], purified by differential preplating, and maintained in Dulbecco's modified Eagle's medium (DMEM) with 10\% (v/v) fetal bovine serum (FBS) in a humidified incubator $\left(37^{\circ} \mathrm{C} ; 95 \%\right.$ air $\left.+5 \% \mathrm{CO} 2\right)$. Myocytes were removed by selective attachment of nonmyocytes to tissue culture dishes during the preplating procedure. Fibroblasts were grown in DMEM supplemented with $10 \%(\mathrm{v} / \mathrm{v})$ FBS and used for the experiments within three passages. As reported previously, the purity of fibroblasts can be consistently maintained at $95 \%$ on the basis of positive immunostaining for vimentin and negative staining for cardiac myosin heavy chain and Von Willebrand factor. 


\section{Cellular Physiology Cell Physiol Biochem 2018;49:1999-2011

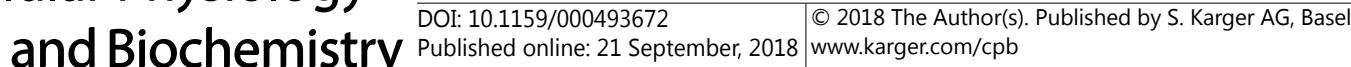 \\ Wang et al.: TSA and Cardiac Remodeling}

Hypoxia treatment in NRCFs and the cell death assay

NRCFs were subjected to hypoxia for $4 \mathrm{~h}$ in vitro in an oxygen control cabinet (Coy Laboratories, Grass Lake, MI) mounted within an incubator and equipped with an oxygen controller and sensor for continuous oxygen level monitoring. A mixture of $95 \%$ nitrogen and $5 \% \mathrm{CO}_{2}$ was utilized to create hypoxia, and oxygen levels in the chamber were monitored and maintained at $<1 \%$, as described previously [26]. Cell death was detected by using the CytoTox 96 Non-Radioactive Cytotoxicity Assay (G1781, Promega Corp. Madison, WI).

\section{Measurement of mitochondrial membrane potential $(\Delta \Psi m)$}

The cultured NRCFs were incubated with tetramethylrhodamine ethyl ester (TMRE, $50 \mathrm{nmol} / \mathrm{L}$, Molecular Probes, Eugene, OR) for $20 \mathrm{~min}$. After loading, the cells were washed twice with Krebs-Henseleit solution. The cells attached to the coverslips were then transferred to a chamber mounted on the stage of a Leica TCS SP2 confocal laser scanning microscope and underwent simulated I/R perfusion. TMRE-loaded cells were excited at a maximum of $543 \mathrm{~nm}$, and the emitted light was collected at 552-620 nm. Twenty cells were randomly selected in each scan using a $\times 20$ objective lens. Images were analyzed using LAS AF Lite software (Leica Microsystems).

\section{Construction of recombinant adenoviruses}

Recombinant adenoviruses expressing short hairpin RNA (shRNA) of LAMP2 (Adsh LAMP2) and LacZ (AdLacZ) were prepared as described previously using the pAdEasy ${ }^{\mathrm{TM}}$ vector system (Qbiogene). Briefly, three short hairpin RNA targeting LAMP2 were cloned into reconstituted pShutle-U6 (Qbiogene) and subjected to homologous recombination in bacteria BJ5183 with pAdeasy-1. The recombinant plasmids were propagated separately in HEK 293 cells. Adenovirus encoding mCherry-green fluorescent protein (GFP)LC3 was purchased from Vigene Bioscience Company, Jinan, China. Adenoviral infection was performed as described previously. After culture of NRCFs for $24 \mathrm{~h}$, adenovirus-directed gene transfer was performed by adding a small volume of FBS-free medium DMEM containing constructed adenovirus at a multiplicity of infection (i.e., the ratio of infectious virus particles to the number of cells being infected) of 50 for $2 \mathrm{~h}$. All experiments were performed after $36 \mathrm{~h}$ of adenoviral infection.

Western blot analysis

The border zones of the infarcted hearts were separated and homogenized. Lysates were prepared in buffer containing $50 \mathrm{mM}$ Tris-HCl (pH 7.4), $150 \mathrm{mM} \mathrm{NaCl}, 1 \mathrm{mM}$ EGTA, $1 \mathrm{mM}$ EDTA, 1\% Triton X-100, and Complete Protease Inhibitor mixture (Roche Applied Bioscience, Mannheim, Germany). Protein concentrations were determined by the Bradford assay using bovine serum albumin standards. Proteins were separated by SDS-PAGE, transferred to nitrocellulose, and immunoblotted with antibodies against LC3 (Sigma-Aldrich, L8918), p62 (Cell Signaling Technology, \#5114), GAPDH (Cell Signaling Technology, \#5174), LAMP2 (Cell Signaling Technology, \#49067), Bcl2 (Cell Signaling Technology, \#2872), and Bax (Cell Signaling Technology, \#14796).

\section{Statistical analysis}

Data are expressed as the mean \pm standard error of the mean. For multiple comparisons, analysis of variance (ANOVA) or repeated ANOVA followed by the least significant difference post-hoc test was used. All analyses were performed using SPSS v13.0 (SPSS Inc., Chicago, IL). A $P$ value $<0.05$ was considered statistically significant.

\section{Results}

TSA treatment led to improved cardiac remodeling and hence cardiac function after MI

To determine the effect of TSA on the progression of cardiac dysfunction, LAD coronary ligation or sham operated surgery was performed in mice that received either TSA treatment or placebo. Eight weeks after MI, LV wall thickness and LV chamber diameter and function were measured by transthoracic echocardiography. MI resulted in significantly decreased cardiac function, as measured by EF, FS, LVPWd, LVPWs, IVSd, and IVSs, and dilatation of the LV chamber as assessed by LVIDd and LVIDs. TSA significantly improved these parameters 


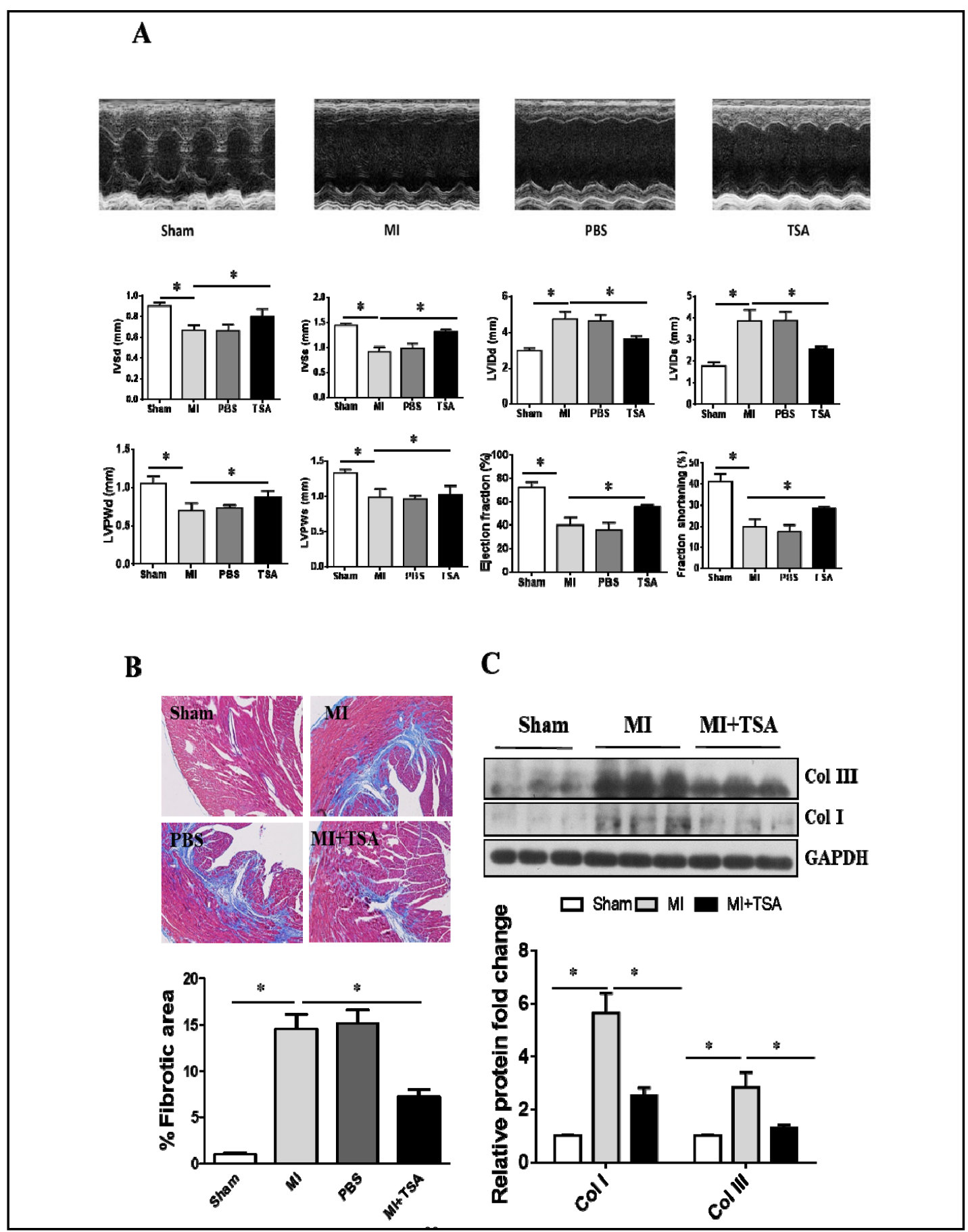

Fig. 1. Histone deacetylase inhibition by TSA augments restoration of ventricular function and prevents cardiac remodeling in mice with MI. A, Representative images (upper panel) and results (lower panel) of echocardiographic assessment of hearts subjected to left anterior descending coronary ligation $(n=5)$. B, Representative and quantitative analysis of Masson trichrome staining of cardiac tissue obtained from the different groups. C, Immunoblot analysis of collagen I and III protein expression in myocardium from the different mouse groups. ${ }^{*} \mathrm{P}<0.05$ vs the indicated group. MI, myocardial infarction; PBS, phosphate-buffered saline; TSA, trichostatin A; IVSd, interventricular septum thickness at end-diastole; IVSs, interventricular septal thickness at end-systole; LVIDd, left ventricular internal diastolic diameter; LVIDs, left ventricular internal systolic diameter; LVPWd, left ventricular diastolic posterior wall thickness; LVPWs, left ventricular systolic posterior wall thickness; Col, collagen.

\section{KARGER}


(Fig. 1A). When cardiac fibrosis was quantified by measuring the LV collagen volume and staining the two fibrotic markers collagen I and III, we found that MI triggered a much greater fibrotic area, which was significantly decreased with TSA treatment (Fig. 1B). The expression levels of both collagen I and collagen III were dramatically increased after MI and significantly decreased by TSA (Fig. 1C). Collectively, these data indicate that TSA improved MI-induced cardiac remodeling.

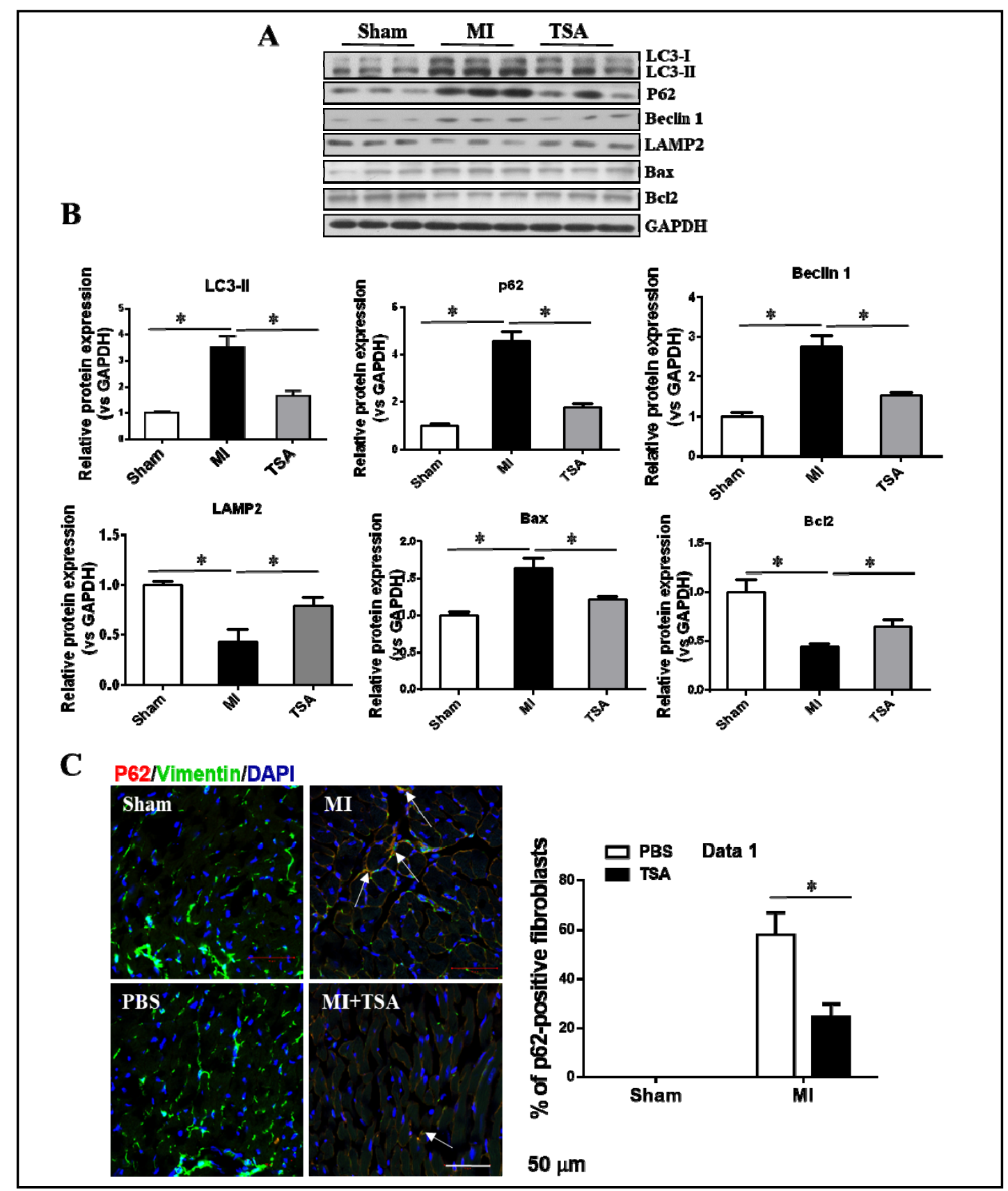

Fig. 2. TSA inhibits MI-induced impairment of autophagosome clearance in vivo. A, Immunoblots and (B) quantitative analysis of LC3, P62, beclin 1, LAMP2, Bcl2 and Bax protein expression in mice myocardium from the different groups $(n=3)$. C, Representative immunofluorescence and quantitative analysis of P62 and the cardiac fibroblast marker vimentin $(n=3) .{ }^{*} \mathrm{P}<0.05$ vs the indicated group. MI, myocardial infarction; TSA, trichostatin A. 
TSA recovered impaired autophagosome clearance after MI

Previous research has demonstrated that ischemic insult (i.e., a brief exposure of myocardial tissue to ischemia) activates cardiomyocyte autophagy, as evidenced by the increased expression ofLC3-II and the numbersofpunctateGFP-LC3-bearingautophagosomes. Similarly, MI significantly increased the expression levels of LC3-II and beclin 1, and these increases were reversed by TSA (Fig. 2A, 2B). Meanwhile, the expression of P62, a specific autophagic substrate protein and hallmark of autophagic flux, was markedly increased after MI but was attenuated by TSA treatment (Fig. 2). Also, the expression of LAMP2, a critical determinant of autophagosome-lysosome fusion, was decreased after MI but was reversed by TSA treatment (Fig. 2A, 2B). Our data suggest that MI impaired autophagosome clearance and that this impairment was alleviated by TSA.

\section{TSA protected NRCFs from hypoxia-induced cell death}

Next, we set out to determine whether the ability of HDACi to suppress pathological cardiac remodeling is cell autonomous. To investigate, primary NRCFs were isolated and exposed to hypoxia in the presence or absence of TSA. Autophagosome accumulation prevents the clearance of damaged intracellular organelles and proteins, leading to increased reactive oxygen species (ROS) generation, followed by diminished mitochondrial membrane potential, which results in mitochondrial permeabilization and the activation of programmed apoptosis and/or necrosis. NRCFs were subjected to hypoxia ( 02 concentration $<1 \%$ ) for $4 \mathrm{~h}$ in vitro. As expected, hypoxia increased ROS generation (data not shown) and decreased the mitochondrial membrane potential, and TSA treatmentreversed the decreased mitochondrial membrane potential after hypoxia (Fig. 3A). Impaired autophagosome processing after ischemic stress caused an accumulation of autophagosomebound LC3-II and p62 (Fig. 2), indicating that impaired autophagosome clearance caused cell death. We further showed that hypoxia-induced NRCF death was inhibited by TSA (Fig. 3B). MI also resulted in a decrease in $\mathrm{Bcl} 2$ and an increase in BAX compared with the sham group, indicating increased apoptotic activity, which was again inhibited by TSA therapy (Fig. 2A). Autophagy dysfunction in $\mathrm{CFs}$ in vivo was further verified by examining the immunofluorescence of P62 in combination with the specific CF marker vimentin. Our results (Fig. 2C) demonstrate that autophagic flux in CFs was impaired after MI and that this was reversed by TSA.

TSA treatment restored autophagic flux after hypoxia exposure

We subjected cultured NRCFs to hypoxia and quantified the relative abundance of autophagosomes and autolysosomes with adenovirus encoding mCherry-GFP-LC3. Adenovirus encoding mCherry-GFP-LC3 helps to distinguish between autophagosomes and autolysosomes. GFP fluorescence

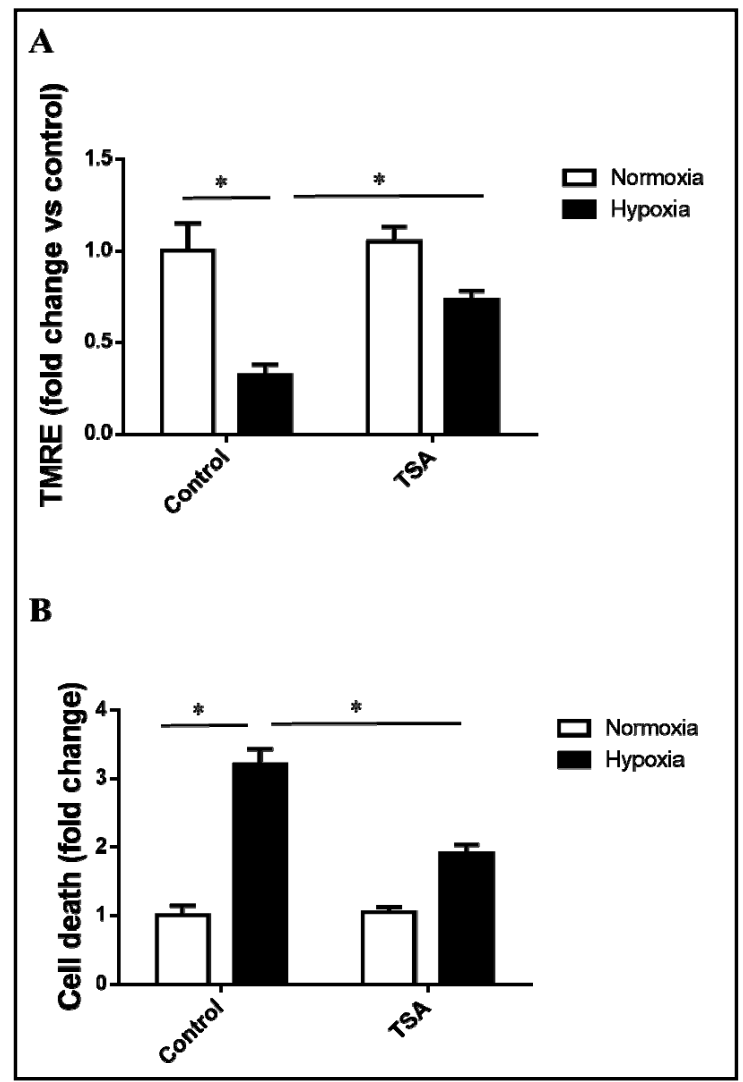

Fig. 3. TSA protects neonatal rat cardiac fibroblasts from hypoxia-induced loss of mitochondrial membrane potential (A) and cell death (B). ${ }^{*} \mathrm{P}<0.05$ vs the indicated group $(n=3)$. TMRE, tetramethylrhodamine ethyl ester; TSA, trichostatin A. 
$\mathbf{A}$
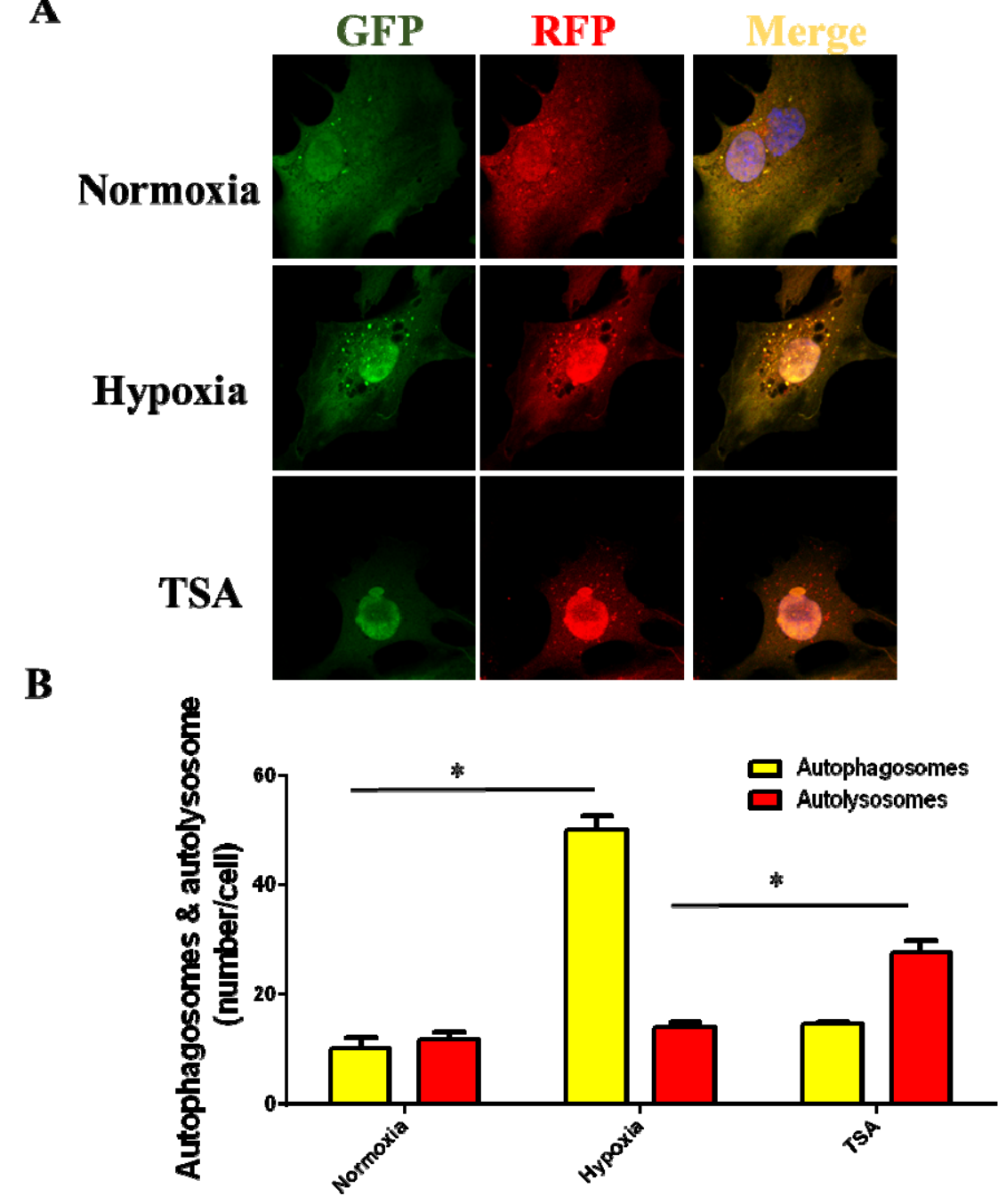

C
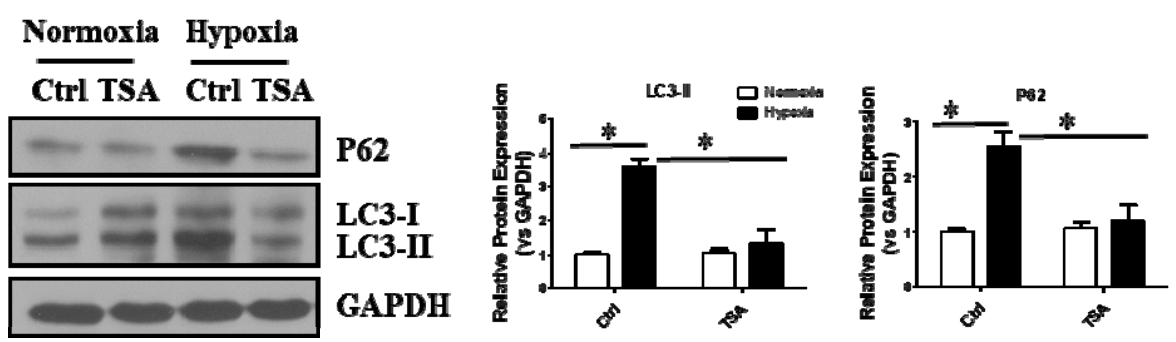

Fig. 4. TSA treatment restores hypoxia-induced impairment of autophagic flux in NRCFs. A, Representative immunofluorescence images demonstrating mCherry-GFP-LC3 localization in NRCFs cultured in a normoxic environment or after hypoxia with or without TSA; representative of 3 experiments. B, Quantitative analysis of autophagosomes (yellow bars) and autolysosomes (red bars) in NRCFs treated as in A ( $\mathrm{n}=25-45$ nuclei per group). C, Representative and quantitative analysis of immunoblots depicting LC3 processing and P62 abundance. ${ }^{*} \mathrm{P}<0.05$ vs the indicated group $(\mathrm{n}=3)$. NRCFs, neonatal rat cardiac fibroblasts; GFP, green fluorescent protein; RFP, red fluorescent protein; TSA, trichostatin A; Ctrl, control. 
is quenched in the acidic $\mathrm{pH}$ of the lysosomal compartment, thereby limiting the use of GFP-LC3 for the identification of autophagosomes. However, mCherry continues to fluoresce, and mCherryLC3 can be used to identify both autophagosomes and autolysosomes. By using mCherry-GFP-LC3 and determining the number of red dots that overlay green dots and appear yellow in merged images, the number of autophagosomes can be evaluated. Red dots that do not overlay green dots and appear red in merged images indicate autolysosome formation. NRCFs cultured in a normoxic environment displayed basal autophagy with intact flux (Fig. 4A). Hypoxia markedly increased the abundance of autophagic structures and increased the numbers of autophagosomes, but there was no increase in the numbers of autolysosomes (Fig. 4B). Treatment of CFs with TSA significantly attenuated hypoxia-induced increases in LC3-II and p62

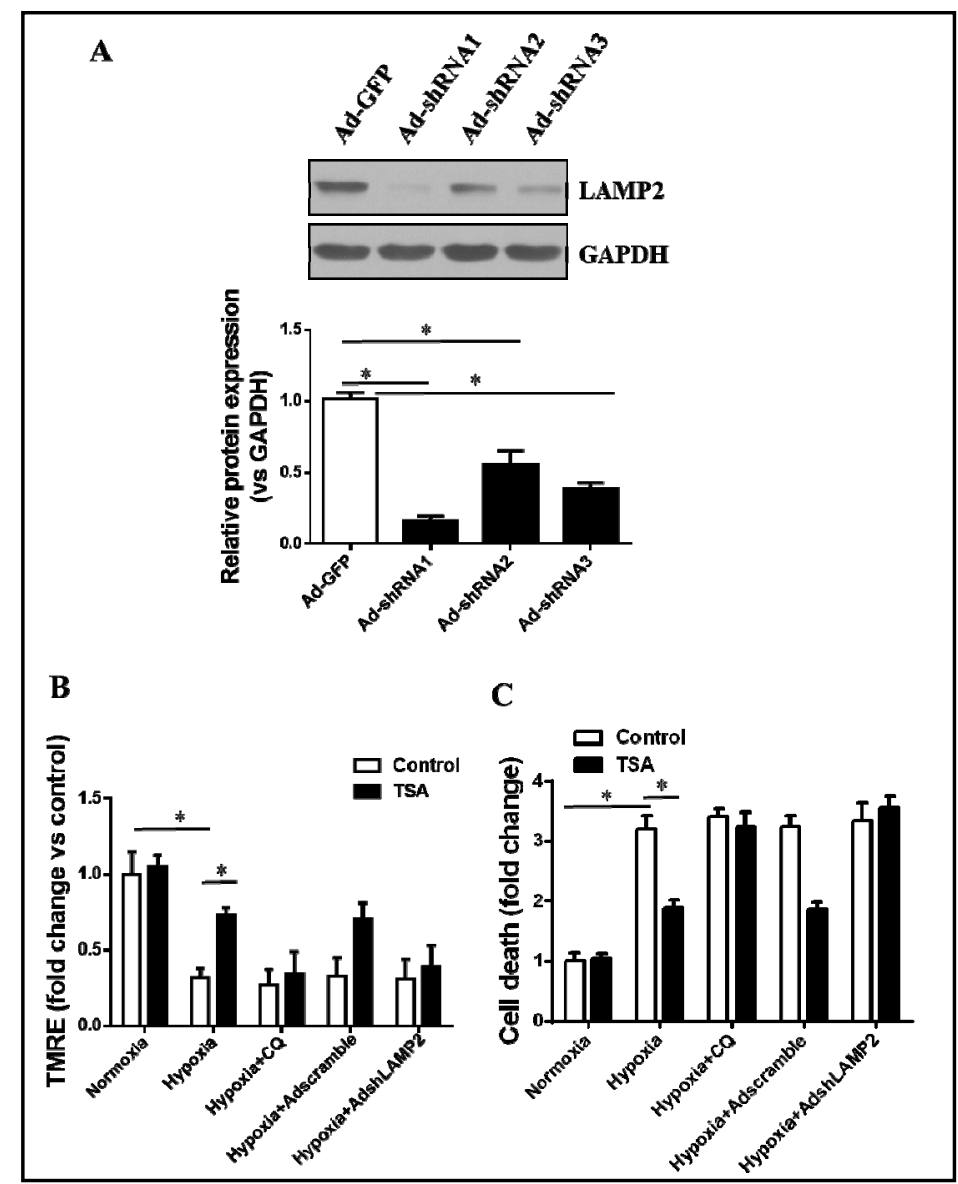

Fig. 5. Restoration of autophagic flux is responsible for the protective effects of TSA against hypoxia-induced cell death. A, Immunoblotting analysis of LAMP2 protein levels. B, Mitochondrial membrane potential (B) and cell death (C) after hypoxia with or without CQ and adenovirus encoding short hairpin RNA of LAMP 2 a. ${ }^{*} \mathrm{P}<0.05$ vs the indicated group $(n=3)$. TMRE, tetramethylrhodamine ethyl ester; TSA, trichostatin A; $\mathrm{CQ}$, chloroquine. (Fig. 4C), and it significantly reduced the number of autophagosomes and increased autolysosome formation, as evaluated by Ad-mCherry-GFPLC3 (Fig. 4A). Together these findings indicate that TSA inhibits ischemic stress-induced autophagy in cardiac myocytes and improves the hypoxia-impaired autophagic flux.

LAMP2 knockdown and chloroquine treatment abolished TSA-mediated protective effects

To investigate whether the restoration of autophagic flux is involved in the cardioprotective effects of TSA, we constructed an adenovirus encoding shRNA for LAMP2, which is a critical determinant of autophagosome-lysosome fusion. Satisfactory knockdown efficiency was obtained at $48 \mathrm{~h}$ post-transfection, as confirmed by western blot (Fig. 5A). We chose the first shRNA for further investigations. We then examined the mitochondrial membrane potential and cell viability after inhibition of autophagic flux via chloroquine (CQ) and LAMP2 gene knockdown, respectively. Our data suggest that CQ and LAMP2 gene knockdowns both abolished TSA-mediated cardioprotection against loss of mitochondrial membrane potential (Fig. 5B) and even cell death (Fig. 5C). Moreover, to assess the involvement of autophagic flux in TSA-mediated cardioprotection, mice were treated with $60 \mathrm{mg} / \mathrm{kg}$ intraperitoneal chloroquine with and without TSA and then subjected to ischemic insult. TSA improved the 


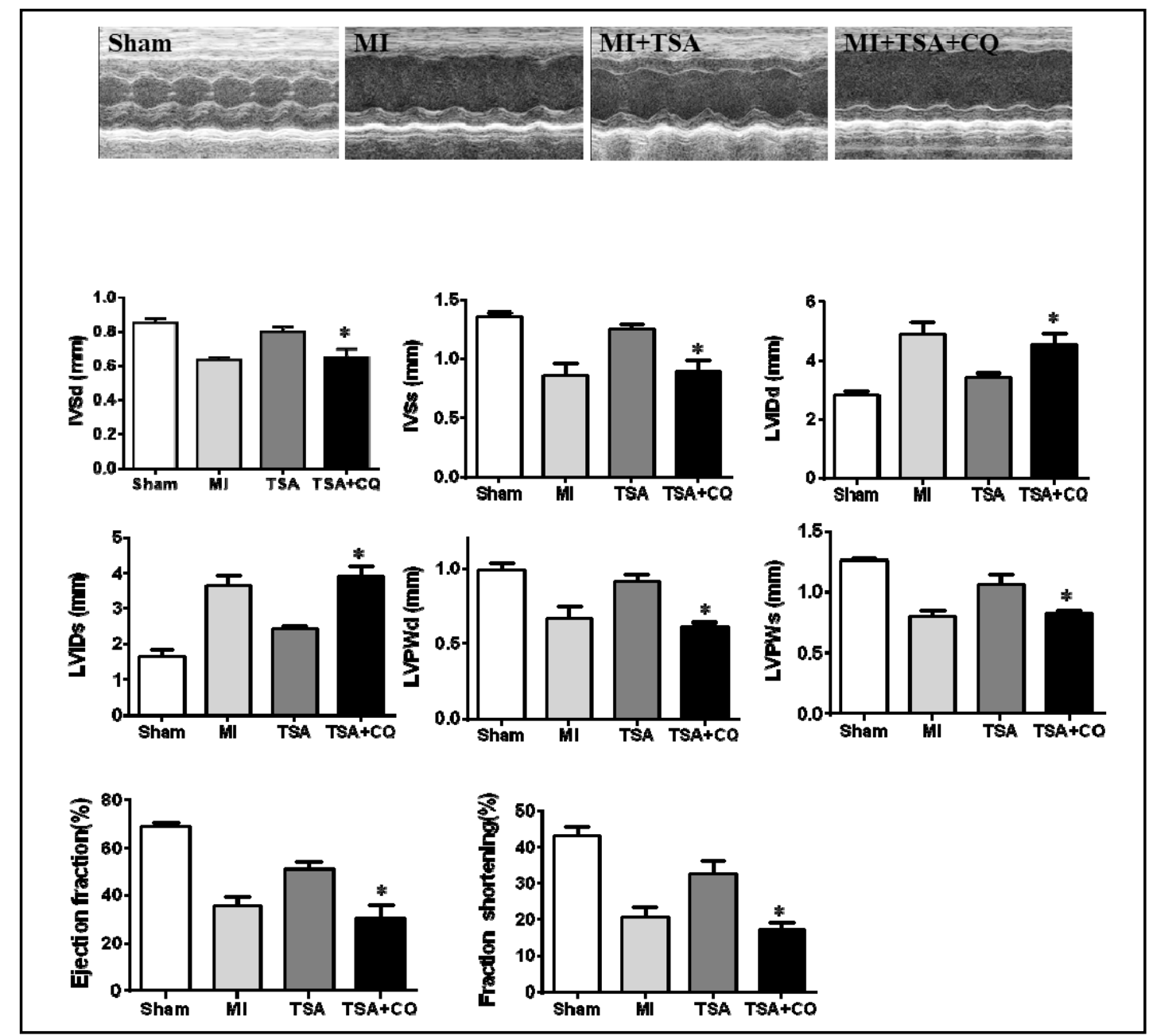

Fig. 6. CQ abolished TSA-improved cardiac functional recovery in MI hearts. Representative images (upper panel) and results (lower panel) of echocardiographic assessment of hearts subjected to left anterior descending coronary ligation. $\mathrm{n}=5,{ }^{*} \mathrm{P}<0.05$ vs the indicated group. CQ, chloroquine; MI, myocardial infarction; PBS, phosphate-buffered saline; TSA, trichostatin A; IVSd, interventricular septum thickness at end-diastole; LVIDs, left ventricular internal systolic diameter.

recovery of cardiac function in MI hearts and this was abolished by chloroquine treatment (Fig. 6). These findings suggest that the HDAC inhibitor TSA attenuates ischemic injury by modulating autophagic flux.

\section{Discussion}

In this study, we demonstrated that 1) autophagosome processing is impaired in CFs undergoing hypoxia treatment and in infarcted hearts; 2) TSA restores autophagosome processing of CFs after hypoxia and in infarcted myocardium; 3) LAMP2 knockdown counteracts TSA-afforded protective effects on CFs; and 4) chloroquine abolishes the TSAinduced cardioprotective effects in MI. These results add to previous findings showing the cardioprotective effects of TSA against MI injury and reveal new mechanisms of TSA for cardioprotection.

Recent work has uncovered the salutary effects of HDAC inhibition in models of heart disease $[27,28]$, and we therefore set out to elucidate the mechanisms of HDAC-dependent 
cardiac plasticity. HDAC activity is required for hypertrophic growth of the myocardium, and the inhibition of HDAC activity can prevent or reverse pathological cardiac remodeling elicited by pressure-overload stress [23]. Moreover, recent studies have demonstrated that HDAC inhibition plays an essential role in the prevention of ischemic injury by the modulation of ventricular remodeling $[6-8,28]$. Consistent with previous findings, our findings show that HDAC inhibition by TSA prevents cardiac modeling after MI and improves the functional recovery of myocardium (Fig. 1). Therefore, TSA treatment may be of benefit to patients with myocardial contractile dysfunction in MI injury.

It is known that autophagy is a hallmark feature of cardiac pathological remodeling and that the administration of HDACi can suppress cardiomyocyte autophagic activity and ameliorate cardiac remodeling [23]. The dynamics and functional role of autophagy during longer term heart ischemia are not clear [29-31]. In the present study, we observed the dynamics of autophagy in infarcted hearts over a longer period after LAD ligation. We found that autophagic proteins LC3-II, P62, and beclin 1 were significantly increased after MI but decreased after TSA treatment in infarcted myocardium (Fig. 2A). Also, the expression of LAMP2, a critical determinant of autophagosome-lysosome fusion, was decreased in MI but reversed by TSA treatment. (Fig. 2B). It appears that MI impaired autophagosome clearance and this was alleviated by TSA.

There have been no previous reports on the effects of HDACi on CFs, which play an important role in cardiac remodeling, and especially on the effects on autophagic activity in CFs after MI. We isolated primary NRCFs and subjected them to hypoxia with and without TSA treatment. Hypoxia markedly increased the abundance of autophagic structures and increased the predominance of autophagosomes with no increase in autolysosomes. Treatment of CFs with TSA significantly attenuated hypoxia-induced increases in LC3-II and p62 accumulation and was accompanied by reduced autophagosome formation and increased autolysosome formation (Fig. 4). Our results demonstrate that TSA inhibited ischemic stress-induced autophagy in CFs and improved the impaired autophagic flux induced by hypoxia. Meanwhile, TSA improved mitochondrial function and cell survival in CFs subjected to hypoxia. Moreover, in vivo manipulation of autophagic flux with CQ abolished the TSA-mediated cardioprotective effects (Fig. 6).

\section{Conclusion}

Although previous studies have shown that TSA has a cardioprotective effect against acute MI and ventricular remodeling, it has not been clear whether TSA inhibits autophagy in CFs in infarcted mouse hearts. This study demonstrated that, at least in the context of MI, HDAC inhibition by TSA modulated the autophagic flux of CFs and blunted cardiac remodeling, thus providing novel mechanisms of HDAC inhibition-induced cardioprotection. Given the ever-expanding burden of MI worldwide, our findings warrant further study in patients with heart disease.

\section{Acknowledgements}

This study was supported grants from the Natural Science Foundation of Zhejiang Province, China (grant no. LY15H020005 to Y.P.W.), the National Natural Science Foundation of China (grant no. 81670235 to Y.P.W.), the Medical Scientific Research Foundation of Zhejiang Province, China (grant no. 2017KY017 to J.F.X.), the Scientific Research Foundation for Excellent Youth Scholars, Zhejiang Provincial People's Hospital, China (grant no. ZRY2016A007 to J.F.X.), and the Advanced Programs for Postdoctoral Fellows of Zhejiang Province, China (grant no. 519000-x81701). We thank Wei Zhu for language assistance during manuscript preparation. 


\section{Cellular Physiology Cell Physiol Biochem 2018;49:1999-2011

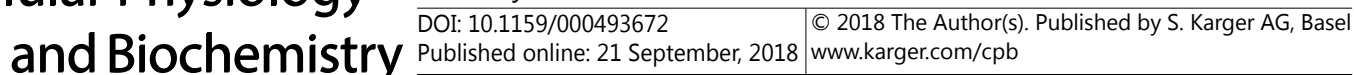 \\ Wang et al.: TSA and Cardiac Remodeling}

\section{Disclosure Statement}

None of the authors have any financial relationships to declare.

\section{References}

1 St. John Sutton MG, Sharpe N: Left ventricular remodeling after myocardial infarction: Pathophysiology and therapy. Circulation 2000;101:2981-2988.

-2 Dirkx E, Gladka MM, Philippen LE, Armand A-S, Kinet V, Leptidis S, el Azzouzi H, Salic K, Bourajjaj M, da Silva GJJ, Olieslagers S, van der Nagel R, de Weger R, Bitsch N, Kisters N, Seyen S, Morikawa Y, Chanoine C, Heymans S, Volders PGA et al.: Nfat and miR-25 cooperate to reactivate the transcription factor Hand2 in heart failure. Nat Cell Biol 2013;15:1282-1293.

-3 Roncarati R, Anselmi CV, Losi MA, Papa L, Cavarretta E, Martins PDC, Contaldi C, Jotti GS, Franzone A, Galastri L, Latronico MVG, Imbriaco M, Esposito G, De Windt L, Betocchi S, Condorelli G: Circulating miR29a, Among Other Up-Regulated MicroRNAs, Is the Only Biomarker for Both Hypertrophy and Fibrosis in Patients With Hypertrophic Cardiomyopathy. J Am Coll Cardiol 2014;63:920-927.

4 Siddesha JM, Valente AJ, Sakamuri SSVP, Yoshida T, Gardner JD, Somanna N, Takahashi C, Noda M, Chandrasekar B: Angiotensin II stimulates cardiac fibroblast migration via the differential regulation of matrixins and RECK. J Mol Cell Cardiol 2013;65:9-18.

5 Yuan ZL, Guan YJ, Chatterjee D, Chin YE: Stat3 dimerization regulated by reversible acetylation of a single lysine residue. Science 2005;307:269-273.

6 Haberland M, Montgomery RL, Olson EN: The many roles of histone deacetylases in development and physiology: implications for disease and therapy. Nat Rev Genet 2009;10:32-42.

-7 Kee HJ, Sohn IS, Il Nam K, Park JE, Qian YR, Yin Z, Ahn Y, Jeong MH, Bang YJ, Kim N, Kim JK, Kim KK, Epstein JA, Kook H: Inhibition of histone deacetylation blocks cardiac hypertrophy induced by angiotensin II infusion and aortic banding. Circulation 2006;113:51-59.

8 Zhao TC, Cheng G, Zhang LX, Tseng YT, Padbury JF: Inhibition of histone deacetylases triggers pharmacologic preconditioning effects against myocardial ischemic injury. Cardiovasc Res 2007;76:473481.

-9 Lee T-M, Lin M-S, Chang N-C: Inhibition of histone deacetylase on ventricular remodeling in infarcted rats. Am J Physiol-Heart C 2007;293:H968-H977.

10 Zhao TC, Zhang LX, Cheng G, Liu JT: gp-91 mediates histone deacetylase inhibition-induced cardioprotection. Biochim Biophys Acta 2010;1803:872-880.

11 Zhang LX, Zhao Y, Cheng G, Guo TL, Chin YE, Liu PY, Zhao TC: Targeted deletion of NF-kappa B p50 diminishes the cardioprotection of histone deacetylase inhibition. Am J Physiol Heart Circ Physiol 2010;298:H2154-H2163.

12 Zhang L, Qin X, Zhao Y, Fast L, Zhuang S, Liu P, Cheng G, Zhao TC: Inhibition of Histone Deacetylases Preserves Myocardial Performance and Prevents Cardiac Remodeling through Stimulation of Endogenous Angiomyogenesis. J Pharmacol Exp Ther 2012;341:285-293.

13 Levine B, Kroemer G: Autophagy in the pathogenesis of disease. Cell 2008;132:27-42.

14 Mizushima N, Levine B, Cuervo AM, Klionsky DJ: Autophagy fights disease through cellular self-digestion. Nature 2008;451:1069-1075.

15 Li Z-L, Lerman LO: Impaired myocardial autophagy linked to energy metabolism disorders. Autophagy 2012;8:992-994.

-16 Kanamori H, Takemura G, Goto K, Maruyama R, Tsujimoto A, Ogino A, Takeyama T, Kawaguchi T, Watanabe T, Fujiwara T, Fujiwara H, Seishima M, Minatoguchi S: The role of autophagy emerging in postinfarction cardiac remodelling. Cardiovasc Res 2011;91:330-339.

17 Hoshino A, Matoba S, Iwai-Kanai E, Nakamura H, Kimata M, Nakaoka M, Katamura M, Okawa Y, Ariyoshi M, Mita Y, Ikeda K, Ueyama T, Okigaki M, Matsubara H: p53-TIGAR axis attenuates mitophagy to exacerbate cardiac damage after ischemia. J Mol Cell Cardiol 2012;52:175-184. 


\section{Cellular Physiology Cell Physiol Biochem 2018;49:1999-2011

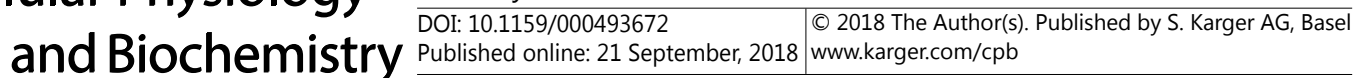 \\ Wang et al.: TSA and Cardiac Remodeling}

18 Kubli DA, Zhang X, Lee Y, Hanna RA, Quinsay MN, Nguyen CK, Jimenez R, Petrosyan S, Murphy AN, Gustafsson AB: Parkin protein deficiency exacerbates cardiac injury and reduces survival following myocardial infarction. J Biol Chem 2013;288:915-926.

19 Ma X, Liu H, Foyil SR, Godar RJ, Weinheimer CJ, Hill JA, Diwan A: Impaired autophagosome clearance contributes to cardiomyocyte death in ischemia/reperfusion injury. Circulation 2012;125:3170-3181.

20 Matsui Y, Takagi H, Qu X, Abdellatif M, Sakoda H, Asano T, Levine B, Sadoshima J: Distinct roles of autophagy in the heart during ischemia and reperfusion: roles of AMP-activated protein kinase and Beclin 1 in mediating autophagy. Circ Res 2007;100:914-922.

-21 Zheng Y, Gu S, Li X, Tan J, Liu S, Jiang Y, Zhang C, Gao L, Yang HT: Berbamine postconditioning protects the heart from ischemia/reperfusion injury through modulation of autophagy. Cell Death Dis 2017;8:e2577.

-22 Yan L, Vatner DE, Kim SJ, Ge H, Masurekar M, Massover WH, Yang G, Matsui Y, Sadoshima J, Vatner SF: Autophagy in chronically ischemic myocardium. Proc Natl Acad Sci U S A 2005;102:13807-13812.

-23 Cao DJ, Wang ZV, Battiprolu PK, Jiang N, Morales CR, Kong Y, Rothermel BA, Gillette TG, Hill JA: Histone deacetylase (HDAC) inhibitors attenuate cardiac hypertrophy by suppressing autophagy. Proc Natl Acad Sci U S A 2011;108:4123-4128.

24 McKinsey TA: Isoform-selective HDAC inhibitors: Closing in on translational medicine for the heart. J Mol Cell Cardiol 2011;51:491-496.

25 Pu J, Yuan A, Shan P, Gao E, Wang X, Wang Y, Lau WB, Koch W, Ma XL, He B: Cardiomyocyte-expressed farnesoid-X-receptor is a novel apoptosis mediator and contributes to myocardial ischaemia/reperfusion injury. Eur Heart J 2013;34:1834-1845.

26 Ma X, Godar RJ, Liu H, Diwan A: Enhancing lysosome biogenesis attenuates BNIP3-induced cardiomyocyte death. Autophagy 2012;8:297-309.

27 Xie M, Kong Y, Tan W, May H, Battiprolu PK, Pedrozo Z, Wang ZV, Morales C, Luo X, Cho G, Jiang N, Jessen ME, Warner JJ, Lavandero S, Gillette TG, Turer AT, Hill JA: Histone deacetylase inhibition blunts ischemia/ reperfusion injury by inducing cardiomyocyte autophagy. Circulation 2014;129:1139-1151.

28 Kong YL, Tannous P, Lu GR, Berenji K, Rothermel BA, Olson EN, Hill JA: Suppression of class I and II histone deacetylases blunts pressure-overload cardiac hypertrophy. Circulation 2006;113:2579-2588.

29 Wu X, He L, Chen F, He X, Cai Y, Zhang G, Yi Q He M, Luo J: Impaired autophagy contributes to adverse cardiac remodeling in acute myocardial infarction. PLoS One 2014;9:e112891.

-30 Thomou T, Mori MA, Dreyfuss JM, Konishi M, Sakaguchi M, Wolfrum C, Rao TN, Winnay JN, Garcia-Martin R, Grinspoon SK, Gorden P, Kahn CR: Adipose-derived circulating miRNAs regulate gene expression in other tissues. Nature 2017;542:450-455.

31 Zhang J, He Z, Xiao W, Na Q, Wu T, Su K, Cui X: Overexpression of BAG3 Attenuates Hypoxia-Induced Cardiomyocyte Apoptosis by Inducing Autophagy. Cell Physiol Biochem 2016;39:491-500. 